\title{
Modeling of Bio-Inspired Self-Repairing Digital System
}

\author{
${ }^{1}$ M.Aarthi, ${ }^{2}$ Dr.S.Karthigailakshmi,M.E,Ph.D., \\ ${ }^{I} M$ E Student, VLSI DesignFrancis Xavier Engineering CollegeTirunelveli, Tamil Nadu \\ ${ }^{2}$ Associate professor,ECE department, Francis Xavier Engineering collegeTirunelveli,Tamil Nadu
}

\begin{abstract}
Self-repairing digital system have recently emerged as the most promising alternative for faulttolerant systems. However, such systems are still impractical in many cases, particularly due to the complex rerouting process that follows cell replacement. They lose efficiency when the circuit size increases, due to the extra hardware in addition to the functional circuit and the un utilization of normal operating hardware for fault recovery. Hence, the system inspired by endocrine cellular communication, that simplifies the rerouting process is proposed. The proposed system is composed of a structural layer and a gene-control layer. The structural layer consists of novel modules and their interconnections. In each module of our system, the encoded data, called the genome, contains information about the function and the connection. Therefore, a faulty module can be replaced and the whole system's functions and connections are maintained by simply assigning the same encoded data to a spare (stem) module. The gene-control layer determines the neighboring spare module in the structural layer to replace the faulty module without collision. The proposed architecture and mechanism are efficient enough for application with real fault-tolerant systems dealing with harsh and remote environments, such as outer space or deep.
\end{abstract}

Index Terms: Bio-inspired engineering, dynamic routing,endocrine cellular communication, redundancy, selfrepair, stem cell.

\section{Introduction}

RELIABILTY has always been an issue with electronic systems ever since the first electronic systems were designed. Different from biological systems, electronic systems are so fragile that even a single problem can render the entire system useless. Therefore, devising fault-tolerant systems that can deal with such delicate problems has been a considerable challenge. During the early stages of the development of fault-tolerant systems, dual modular redundancy (DMR) and triple modular redundancy (TMR) methods were introduced . These techniques ran the same modules in parallel, and thus a faulty module could be distinguished by comparing outputs of the same modules and voting for the majority one (with TMR) or by using an additional device (with DMR). However, these methods have several problems. The size of the module is so huge that a large part of the circuit must be replaced even if a small part in the module is malfunctioning. Furthermore, redundancy has to be running all the time, and it can only cover the fault once.

Each chip contains more than 100,000 transistors (the most basic component in a microchip), which don't all function simultaneously. Rather, the researchers burned vast swaths of transistors out of the chip with a laser, then allowed the systems to recalibrate. As long as the blast did not catch any data caches in its crosshairs, the chip could seek out alternate routes and continue to function. With the help of an applicationspecific integrated-circuit (ASIC) processor on each chip, the system could "learn" which pathways were broken and adjust accordingly.But, rerouting is complex and collapses the entire circuit. In the last 10 years, these conventional methods have proven to be rather inefficient, and scientists have consequently turned to biology to find inspiration for a more suitable self-repairing circuit that can resolve the aforementioned problems and faults with fault-tolerant systems.This project get inspiration from bio cell-cell communication for designing self repairing circuit. The study of cell communication focuses on how a cell gives and receives messages with its environment and with itself. Indeed, cells do not live in isolation. Their survival depends on receiving and processing information from the outside environment, whether that information pertains to the availability of nutrients, changes in temperature, or variations in light levels. Cells can also communicate directly with one another and change their own internal workings in response by way of a variety of chemical and mechanical signals.In multicellular organisms, cell signaling allows for specialization of groups of cells. Multiple cell types can then join together to form tissues such as muscle, blood, and brain tissue. In single-celled organisms, signaling allows populations of cells to coordinate with one another and work like a team to accomplish tasks no single cell could carry out on its own. The study of cell signaling touches multiple biological disciplines, such as developmental biology, neurobiology, and endocrinology. Consequently, the relevance of cell communication is quite vast, but major areas of fundamental research are often divided between the study of signals at the cell membrane and the study of signals within and between intracellular compartments. Membrane signaling involves proteins shaped into receptors embedded in the cell's membrane that biophysically connect the triggers in the external environment to the ongoing dynamic chemistry inside a cell. Signaling at the membrane also 
involves ion channels, which allow the direct passage of molecules between external and internal compartments of the cell. Cells have evolved a variety of signaling mechanisms to accomplish the transmission of important biological information. Some examples of this variety are receptors that allow ion currents to flow in response to photons, which effectively translates light into chemical messengers inside the cone and rod cells of the retina; growth factors that interact with the cell membrane and can trigger receptors that powerfully affect chromatin structure and the modulation of gene expression; metabolites in the blood that can trigger a cell's receptors to cause the release of a hormone needed for glucose regulation; adhesion receptors that can convey tensiongenerated forces that direct a cell to stay put or change direction of movement; and developmentally regulated receptors that can strictly guide the path of a migrating cell, ultimately controlling how an entire organism is wired together. How do scientists go about studying such an intricate meshwork of interactions at the crossroads of chemistry, physics, and biology? One method is reductionist, whereby cells are isolated and cultured in vitro so that specific signals can be carefully tested with chemicals and cellular responses can be measured. Another more holistic method involves measuring cellular signaling in an intact organism (in vivo) by applying specific chemical agents that block or activate receptors in a carefully chosen tissue region and then measuring the response via an electrode that relays the activity of ion currents or via fluid sampling of the activated area. For both approaches, response measurement is vitally important, and measuring the small cellular entities is indeed a challenge.Scientists use sophisticated time-lapse microscopy to track labeled molecules that travel between subcellular compartments after a signaling event or to track the conformation of a receptor that has gone from an inactive to an active state. Furthermore, mass spectrometry techniques permit measurements of picomolar amounts, enabling the tracking of intracellular second messenger molecules that are crucial in the regulation of signals in the intracellular milieu.Despite technical advances, global understanding of signal transduction, its internal hierarchies, and its highly integrated and extremely dynamic nature remains largely mysterious.A potential breakthrough in the field arose recently when scientists realized that there are striking analogies between signaling networks in biological systems and electronic circuits; both of them involve hierarchies, switches, modularity, redundancy, and the existence of powerful feedback mechanisms. Such a realization gave impetus to the field of computational biology as applied to cellular signaling.Today, the study of cell signaling is not restricted to biologists; with the contribution of engineers and biophysicists, scientists can now create computational algorithms that model the structure of a signaling network based on biological measurements, and these models can be used to predict the outcome of otherwise physically impossible experimental conditions. As it turns out, we are just beginning to appreciate that many of the designs and strategies we have developed to manipulate information, particularly within the digital world, are actually present in biological networks, having already been invented over the course of a hundred million years of evolution.

An approach called endocrine cellular system is the application of conceptsinspired from the biological cell to the design of digital circuits. As the biological cells carry the genetic code of the whole system and are differentiated according to the location of the cell in the system, an embryonic self-repairing circuit is organized with building blocks that have identical structures and that vary according to the expressed genetic code in each block. These self-repairing circuits can also recover from a fault by isolating the faulty block and differentiating a spare (stem) block with the same genetic code previously held by the faulty block. With such biological inspiration, self-repairing systems make repairs on a fine-grained scale rather than the coarse-grained scale of conventional fault tolerant systems. Thus, such systems only need to change a small part of the system, and the spare (stem) blocks do not need to operate constantly. As an additional advantage, spare (stem) blocks can become any kind of logic, such as a biological stem cell (SC). Moreover, these systems can recover a functional cell (module) several times, whereas conventional fault-tolerant systems deal with only a single fault. "Bringing this type of electronic immune system to integrated-circuit chips opens up a world of possibilities."

\section{New Self-Repairing Mechanism from Endocrine Cellular Communication}

In this project, the proposed system simplifies the self-repairing mechanism and helps the circuit to maximize the efficiency of its hardware realization. The size of the hardware can grow in proportion to the number of functional modules while also ensuring good fault-coverage. The proposed self-repairing system is inspired by the endocrine cellular communication System, Where in a special endocrine cell secretes a specific hormone only after it receives another specific hormone. Each hormone flows in the blood vessel that is adjacent to all the endocrine cells. Thus, the blood vessel maintains the connection among endocrine cells, even when the endocrine cell is replaced by an SC that differentiates into a cell having the same gene as the dead cell. As a result, a new endocrine cell can receive and secrete the same hormones as did previous cells through the blood vessel. By adopting a similar mechanism in an electronic circuit, we devised a system having a novel encoding strategy in each module and also a novel wiring architecture that can maintain the function as well as connections by replacing the faulty module with a spare (stem) module without any additional rerouting process.

The proposed system architecture is composed of a structural layer and a gene-control layer. In the structural layer, the circuit is divided into LUT-based modules. Encoded data in each module are composed of 
both functional and connection data, and a new wiring architecture between modules is also developed. Therefore, the function and connections of the whole system are maintained by simply expressing the same encoded data to the spare (stem) module, while the wiring architecture connects correctly after the encoded data are properly assigned to the spare module. There are already several available devices that can replace a faulty module by embedding the same encoded data of the faulty module into the stem module, but none of them can maintain the function and connections through single-step embedding of the encoded data into the spare module, as proposed in this paper.

The fault signal is generated in the structural layer and then reaches the gene-control layer, which determines the spare (stem) module in the structural layer that will take the faulty module instead. The main role of the genecontrol layer is to assign the correct spare (stem) module to replace the faulty one. In addition, modules involved in this mechanism are distributed and operated in parallel.Therefore, even if several faults occur simultaneously in different modules, the system can recover them. Furthermore, it can preserve the state and function that existed just before the fault occurred in the sequential system.

\section{A. Endocrine Cellular System}

The endocrine system is referring to the collection of cells, glands, and tissues of an organism that secretehormones
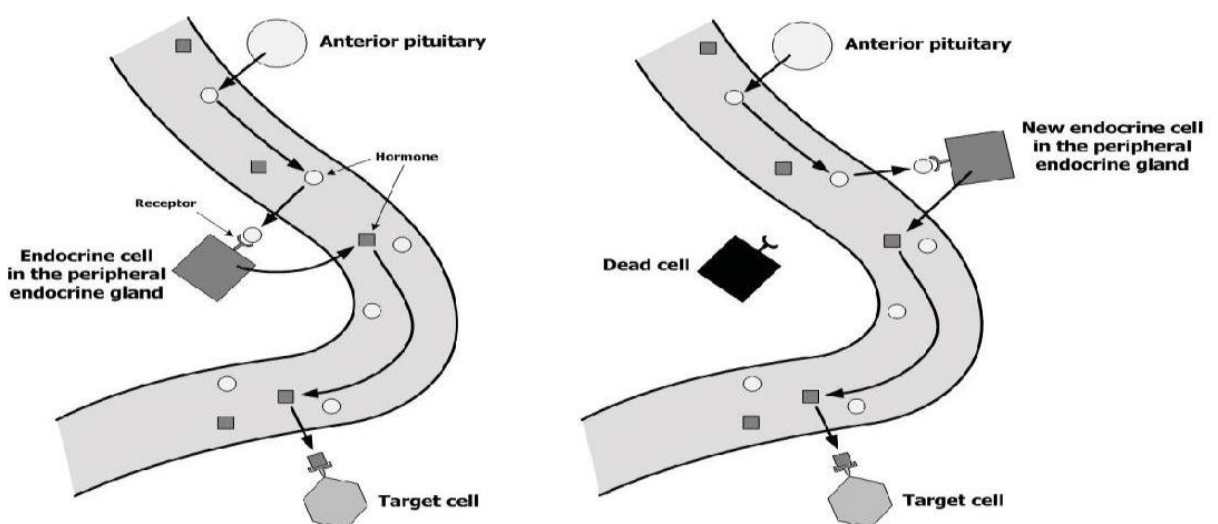

Fig. 1.Special endocrine cellular communication. (a) Normal state.

Directly into the bloodstream (some of which are transported along nerve tractsto control the organisms' physiological and behavioral activities. The endocrine system is in contrast to the exocrine system, which secretes its chemicals using ducts. The word endocrine derives from the Greek words "endo" meaning inside, within, and "crinis" for secrete. The endocrine system is an information signal system like the nervous system, yet its effects and mechanism are classifiably different. The endocrine system's effects are slow to initiate, and prolonged in their response, lasting from a few hours up to weeks. The nervous system sends information very quickly, and responses are generally short lived. Hormones are substances (chemical mediators) released from endocrine tissue into the bloodstream where they travel to target tissue and generate a response. Hormones regulate various human functions, including metabolism, growth and development, tissue function, sleep, and mood. Features of endocrine glands are, in general, their ductless nature, their vascularity, and usually the presence of intracellular vacuoles or granules storing their hormones. In contrast, exocrine glands, such as salivary glands, sweat glands, and glands within the gastrointestinal tract, tend to be much less vascular and have ducts or a hollow lumen.In addition to the specialised endocrine organs mentioned above, many other organs that are part of other body systems, such as the kidney, liver, heart and gonads, have secondary endocrine functions. For example the kidney secretes endocrine hormones such as erythropoietin and renin. The endocrine system is made of a series of glands that produce chemicals called hormones. A number of glands that signal each other in sequence are usually referred to as an axis, for example, the hypothalamic-pituitary-adrenal axis.

\section{(b) Recovery after cell apoptosis.}

\section{B.New Self-Repairing Mechanism From Endocrine Cellular Communication}

Among the various methods of cell-to-cell communication in our body, endocrine cellular communication is particularly interesting. Basically, an endocrine cell releases a hormone from the signaling endocrine cell, and the hormone flows through the blood vessel until it binds to the target cell. Although the blood contains various hormones, only the receptor on the target cell receives the selected hormone. The special method of endocrine cellular communication that inspired this project is based on a specific endocrine cell that secretes a hormone only if it receives another hormone from another endocrine cell . 
The hormone from the anterior pituitary stimulates the endocrine cell in the peripheral endocrine gland to secrete the peripheral gland hormone. As a concrete example, corticotropin enters the blood and travels tothe adrenal cortex where it stimulates the release of cortisol. Thus, the blood vessel delivers the hormones between cells. When a functioning endocrine cell dies through apoptosis, the special endocrine cellular communication maintains hormone delivery by differentiating an SC into a cell having the same genome part as that of the dead cell [Fig.4.1]. Thus, in addition to its own functioning, the genome in the endocrine cell has the information about connections between cells. Cell signalling is part of a complex system of communication that governs basic cellular activities and coordinates cell actions.

\section{Self -Repairing Architecture}

The inspiration that obtained from the biological endocrinesystem lies in the efficient and flexiblecommunication mechanism between endocrine cells. In the endocrine system, the information between endocrine cells is exchanged via the hormones, and this forms a complex
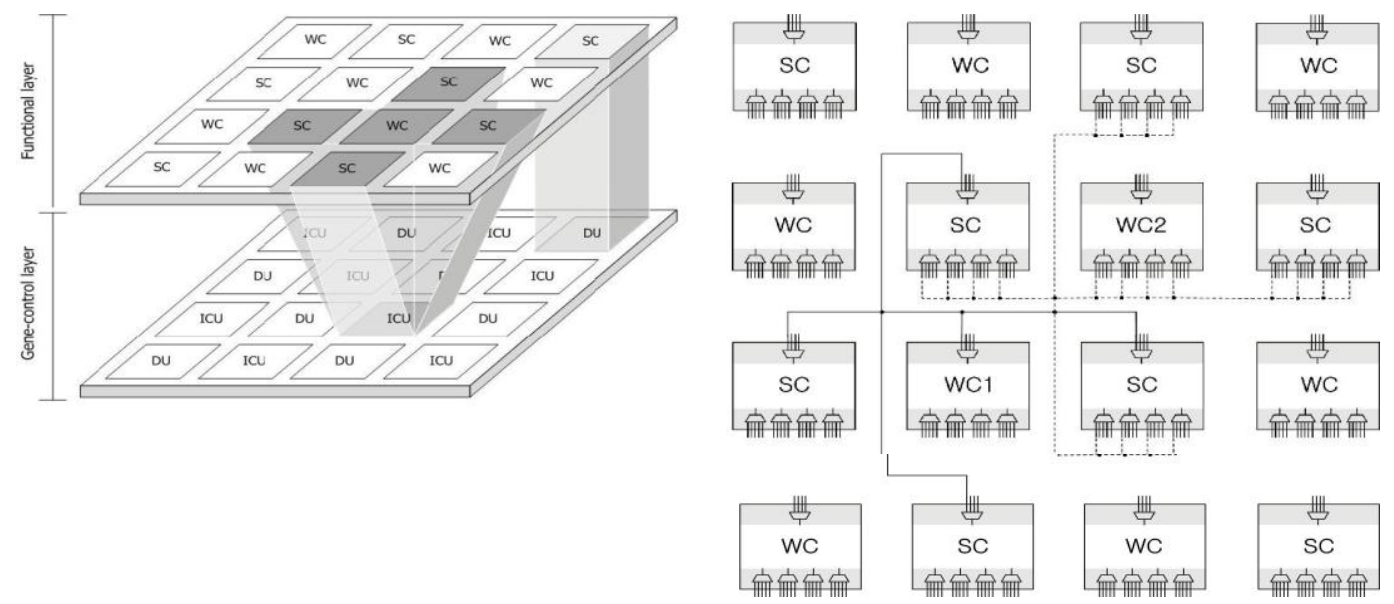

Fig. 2.Proposed self-repairing digital system.Overall architecture and related cells between the functional layer and the control layer. WC: working cell. SC: stem cell. ICU: index changing unit. DU: differentiation unit and Artificial endocrine routing architecture, the proposed connection froWC1 to WC2 by a connecting solid line and dotted line Solid line: the proposed connection for the output of WC1. Dotted line: the proposed connection for the input of WC2.

Communication network. The structure of this network is flexible and easily changed by choosing and adjusting the hormones to be used for secretion and reception in each endocrine cell. Even if an endocrine cell dies through apoptosis, a new endocrine cell having the same function of the dead cell is produced by differentiation and the overall communication network is recovered. Inspired from this feature of the endocrine system,the proposed system has been developed. In particular, to implement an electronic module that resembles the featureof an endocrine cell receiving and secreting the hormones selectively, multiplexers (MUXs) for selection of inputs and demultiplexers (DEMUXs) for selection of outputs have been introduced.In order to separate the roles of hardware structure and control, this project propose a functional layer and a gene-control layer, as depicted in Fig 4.2.

The functional layer consists of an artificial endocrine cell (module) and the artificial endocrine routing architecture between cells. The artificial endocrine cell has a basic structure, genome (encoded data), and fault detection unit. The structure of each cell is identical and modules are classified as working, stem (spare), or isolated cells. The only difference between the cells is the genome in the cell. Every working cell (WC) has four neighboring SCs

And the WC can be replaced by any available SC among them in the event of fault occurrence. In the gene-control layer, the index changing unit (ICU) takes charge of one WC and its four neighboring SCs in the functional layer. It chooses the proper candidate SC for the faulty WC without collision. The differentiation unit (DU) differentiates (reprograms) the SC when the ICU chooses the SC as an alternative to the faulty WC. 
WCs in the proposed system can be expanded to any direction as far as every WC has four neighboring SCs. Because the SC can be used for four nearby WCs by exact control without collision. A WC can be recovered up to four times even though the number of SCs is similar to the number of WCS .

\section{D.New Self-Repairing Architecture: Functional Layer E.Artificial Endocrine Cell: Basic Structure And Genome}

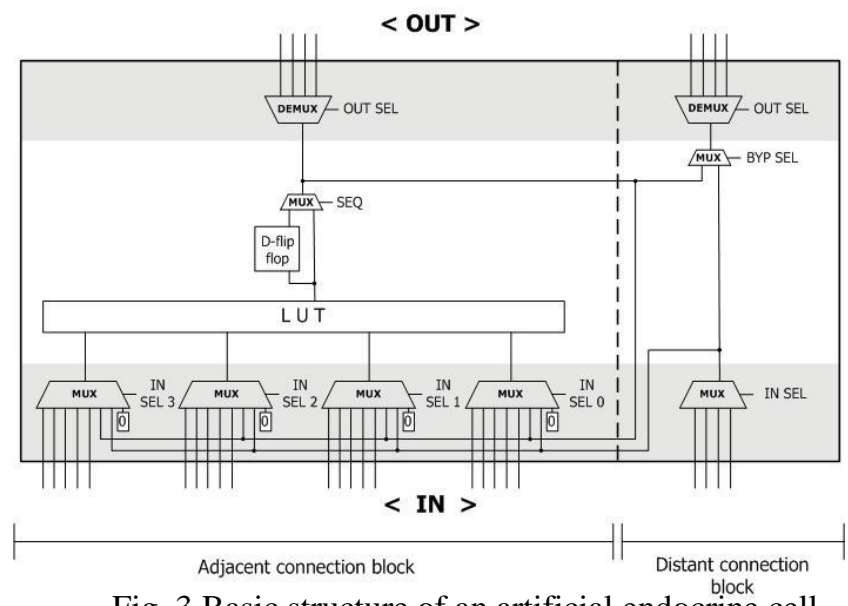

Fig. 3.Basic structure of an artificial endocrine cell.

The basic structure of the proposed artificial endocrine celloperates on the basis of the four-input LUT (Fig 4.3). It also has a D-type flip-flop for the sequential circuit, the MUX, and the DEMUX. The left block of the basic structure connects the cell to the adjacent cells whereas the right block connects to distant cells. In the left block, inputs of four MUXs are connected to the adjacent cells, its output value, the right block, and the fixed value. Four inputs of the LUT yield the output based on the LUT, and flip-flop can be used by choice for sequential behavior. The output in the left block can also be transmitted to the right block for a distant connection. The outputs of DEMUX are also connected to other cells. In the right block, the signal from the distant cell is connected to the left block for the input of the LUT or it bypasses the cell. Table 4.1 summarizes the genome of the artificial endocrine cell, which is implemented in the form of a register. The genome exists in every WC and SC and consists of selection bits of MUX, DEMUX, and LUT bits. Because the whole application system operates with the assembly of WCs and the operation of each cell is based on the genome, the genome plays the most important role in the cell. Inspired from this feature of the endocrine system, the proposed system has been developed. In particular, to implement an electronic module that resembles the feature of an endocrine cell receiving and secreting the hormones selectively, multiplexers (MUXs) for selection of inputs and demultiplexers (DEMUXs) for selection of outputs have been introduced . It is also noted that the hormone which is selectively received and secreted by the endocrine cell is determined by the genome that is expressed for differentiation of an SC. The above table shows the genome which is the encoded data for fault recovery.It is divided in to adjacent and distant block cells. The inputs and out puts for both the block is given in the table.

\section{F.Artificial Endocrine Routing Architecture}

To realize the routing architecture, the WCs and SCs are arranged as shown in Fig. 4.4.In Fig. 4.4, if the output of WC1is connected to the input of WC2 while two WCs can be replaced by neighboring SCs, two types of routing must be made above all. WC1, which sends the output, is connected to four neighboring SCs (the solid line in Fig. 4.4) while WC2, which receives the output of WC1 as its input, is connected in the same way (the dotted line in Fig. 4.4). For each type of line in Fig. 4, the same order of inputs of MUXs among cells must be connected. Then, two types of wires arconnected.The distant connection block (Fig. 4.4), which is not illustrated in Fig. 4.4, has the same types of connections. As shown in Fig. 4, the proposed routing architecture is composed of connection wires and input selection MUXs in order to dynamically connect the output of WC1 (or the SCs of WC1) to the input of WC2 (or the SCs of WC2). The real connection among inputs and the outputs of the cells are controlled by the genome of each cell. For example, if the output of WC1 is initially connected to the input of WC2through the first MUX in WC2, all of the other inputs and outputs of cells that are connected to the wire are disconnected.

The outputs of SCs are blocked such that they do not interfere with other operating cells. After WC2 substituted the SC that is located on the right side of WC2, the SC can have the same connection as that of WC2 
before the fault occurrence only by expressing the genome of WC2 to the SC and isolating WC2,because the same order of the inputs of MUXs between WC2 and the SC are connected and the genome has the selection bits of MUXs.

TABLE 1GENOME(ENCODED DATA) IN THE ARTIFICIAL ENDOCRINE
\begin{tabular}{l|l|l|}
\multirow{4}{*}{ Adjacent connection block } & Input and input selection & $\begin{array}{l}{[19: 0]} \\
\&[11: 0]\end{array}$ \\
\cline { 3 - 4 } & Output demux selection & {$[1: 0]$} \\
\cline { 2 - 3 } & Lookup table & {$[4: 1]$} \\
\cline { 2 - 3 } & Flip flop selection & Seq \\
\hline Distant connection block & $\begin{array}{l}\text { Input and input MUX } \\
\text { selection }\end{array}$ & {$[3: 0]$} \\
\cline { 2 - 4 } & $\begin{array}{l}\text { Output DEMUX } \\
\text { selection }\end{array}$ & {$[1: 0]$} \\
\cline { 2 - 3 } & Byepass & Byesel \\
\hline
\end{tabular}

\section{Results and Dicussion}

The working of artificial endocrine cell has been realized using even parity.The results is based on even no of ' 1 's.Here 20 bits is given as input.the output is based on the selection of mux and LUT produces the output based on parity.By creating a cell pattern the system is repaired manually. The results are simulated using Xilinx ise 12.2i.

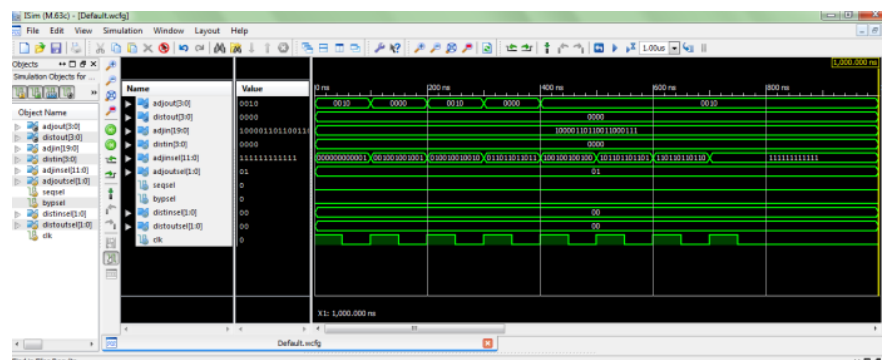

Fig 4 simulation result of artificial endocrine cell.

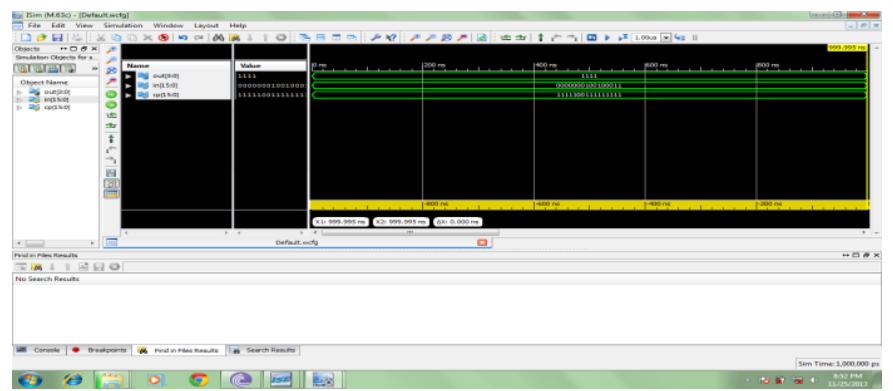

Fig 5 simulation result of cell replacement

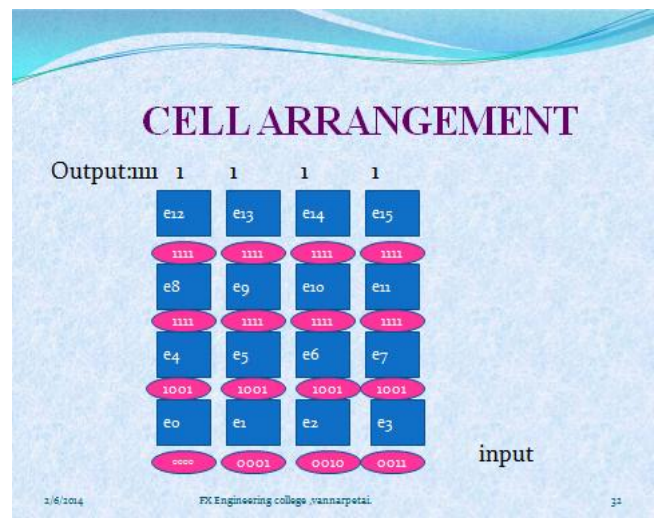

(a) 


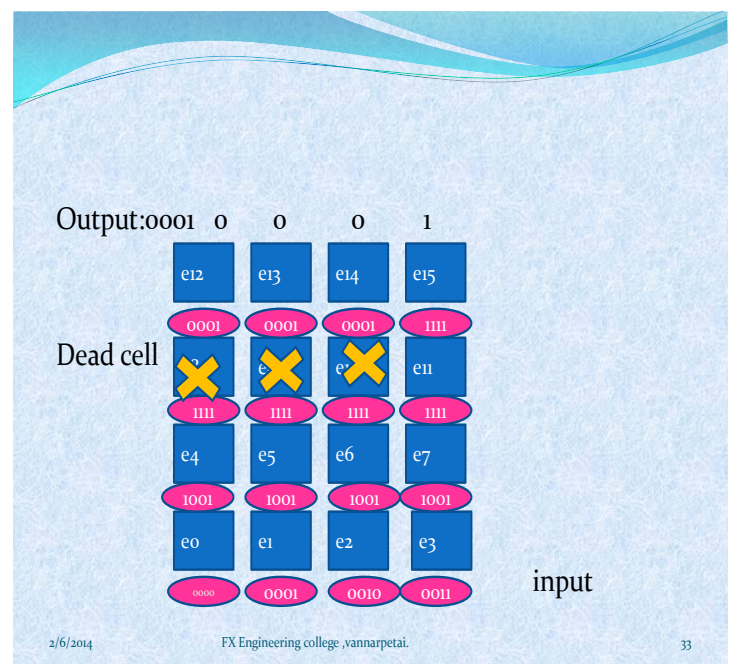

(b)

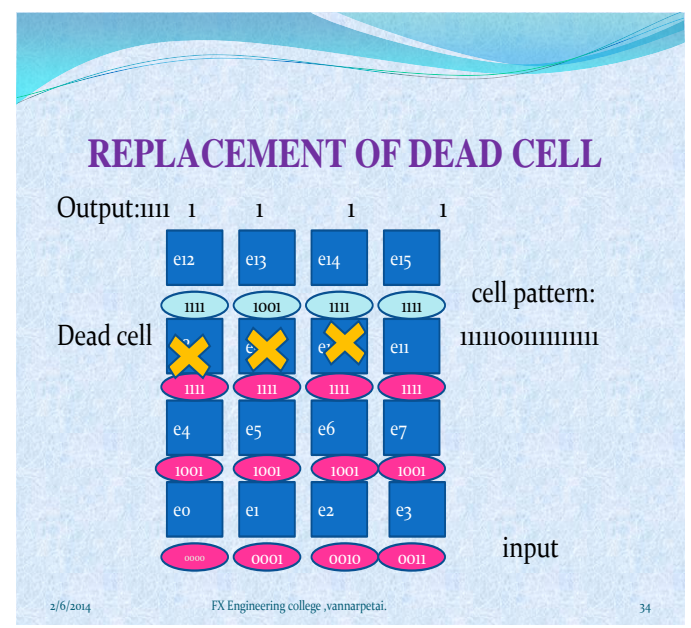

(c)

Fig 6 (a) Cell arrangement (b) faulty cell (c) creation of cell pattern

The fig 6 shows the manual replacement of dead cell.thus I have tested the artificial endocrine cell.

\section{Conclusion}

In this paper, a new self-repairing digital system consists of a functional layer and a gene-control layer was proposed. The functional layer performs the function and the gene-control layer controls the functional layer so that proper assignment of SC for the replacement of a faulty cell is carried out. New architectures for the routing and the working of artificial endocrine cell were studied.The results of the functional layer were also simulated. For further improvement of the proposed self-repairing system, the new protocol for index control unit in gene control layer for self-repairing digital system should be further investigated in a future study.

\section{References}

[1] W. C. Carter, "Fault-tolerant computing: An introduction and a viewpoint,"IEEE Trans. Comput., vol. 22, no. 3, pp. 225-229, Mar. 1973.

[2] C. Ortega and A. Tyrrell, "Design of a basic cell to construct embryonicarrays," IEE Proc. Comput. Digital Tech., vol. 145, no. 3, pp. 242-248,May 1998.

[3] X. Zhang, G. Dragffy, A. G. Pipe, N. Gunton, and Q. M. Zhu, "Areconfigurable self-healing embryonic cell architecture," in Proc. ERSA,Jun. 2003, pp. 134-140.

[4] D. Mange, E. Sanchez, A. Stauffer, G. Tempesti, P. Marchal, andC. Piguet, "Embryonics: A new methodology for designing fieldprogrammablegate arrays with self-repair and self-replicating properties,"IEEE Trans. Very Large Scale Integr. (VLSI) Syst., vol. 6, no. 3,pp. 387-399, Sep. 1998.

[5] D. Mange, S. Durand, E. Sanckez, A. Stauffer, G. Tempesti, P. Marchal,and C. Piguet, "A new paradigm for developing digital systems based ona multi-cellular organization,” in Proc. IEEE Int. Symp. Circuits Syst.,vol. 3. Apr.-May 1995, pp. $2193-2196$.

[6] W. Barker, D. M. Halliday, Y. Thoma, E. Sanchez, G. Tempesti, andA. M. Tyrrell, "Fault tolerance using dynamic reconfiguration on thePOEtic tissue,” IEEE Trans. Evol. Comput., vol. 11, no. 5, pp. 666-684,Oct. 2007. 
[7] P. K. Lala and B. K. Kumar, “An architecture for self-healing digitalsystems,” J. Electron. Testing: Theory Appl., vol. 19, no. 5, pp. 523-535, Oct. 2003.

[8] M. Samie, G. Dragffy, A. Popescu, T. Pipe, and C. Melhuish, "Prokaryoticbio-inspired model for embryonics," in Proc. NASA/ESA Conf.Adapt. Hardw. Syst., Jul.-Aug. 2009, pp. 163-170.

[9] M. Samie, G. Dragffy, A. Popescu, T. Pipe, and J. Kiely, "Prokaryoticbio-inspired system," in Proc. NASA/ESA Conf. Adapt. Hardw. Syst.,Jul.-Aug. 2009, pp. 171-178.

[10] M. Samie, G. Dragffy, and T. Pipe, "Bio-inspired self-test for evolvablefault tolerant hardware systems," in Proc. NASA/ESA Conf. Adapt.Hardw. Syst., Jun. 2010, pp. 325-332.

[11] M. Samie, G. Dragffy, and T. Pipe, "UNITRONICS: A novel bioinspiredfault tolerant cellular system," in Proc. NASA/ESA Conf. Adapt.Hardw. Syst., Jun. 2011, pp. 58-65.

[12] D. Mange, M. Sipper, A. Stauffer, and G. Tempesti, “Toward robustintegrated circuits: The embryonics approach,” Proc. IEEE, vol. 88,no. 4, pp. 516-541, Apr. 2000.

[13] G. Tempesti, "A self-repairing multiplexer-based FPGA inspired bybiological processes," Ph.D. dissertation, Dept. Comput. Eng., PrincetonUniv., Princeton, NJ, 1998.

[14] J. R. Heath, P. J. Kuekes, G. S. Snider, and R. S. Williams, "A defecttolerantcomputer architecture: Opportunities for nanotechnology," Science, vol. 280, pp. 1716-1721, Jun. 1998.

[15] L. J. K. Durbeck and N. J. Macias, "Defect-tolerant, fine-grained paralleltesting of a cell matrix," Proc. SPIE, vol. 4867, no. 8, pp. 71-85, Jul.2002.

[16] J. Lohn, G. Larchev, and R. Demara, "A genetic representation forevolutionary fault recovery in virtex FPGAs," in Lecture Notes inComputer Science. New York: Springer-Verlag, 2003.

[17] P. K. Lala, B. K. Kumar, and J. P. Parkerson, "On self-healing digitalsystem design,” Microelectron. J., vol. 37, no. 4, pp. 353-362, Apr. 2006.

[18] J.-M. Moreno, Y. Thoma, E. Sanchez, O. Torres, and G. Tempesti, "Hardware realization of a bio-inspired POEtic tissue," in Proc.NASADoD Conf. Evolvable Hardw., 2004, pp. 237-244.

[19] N. J. Macias and L. J. K. Durbeck, "Adaptive methods for growingelectronic circuits on an imperfect synthetic matrix," Biosystems, vol. 73,no. 3, pp. 173-204, Mar. 2004.

[20] Y. Thoma, E. Sanchez, J.-M.Moreno, J. Arostegui, and G. Tempesti, “Adynamic routing algorithm for a bio-inspired reconfigurable circuit," inLecture Notes in Computer Science. New York: Springer-Verlag, 2003,pp. 681-690.

[21] A. J. Greensted and A. M. Tyrrell, "An endocrinologic-inspired hardwareimplementation of a multicellular system," in Proc. NASA/DoD Conf.Evolvable Hardw., 2004, pp. 245-252.

[22] A. J. Greensted and A. M. Tyrrell, "Implementation results for a faulttolerantmulticellular architecture inspired by endocrine communication,"inProc. NASA/DoD Conf. Evolvable Hardw., 2005, pp. 253-261.

[23] B. Alberts, A. Johnson, J. Lewis, M. Raff, and K. Roberts, MolecularBiology of the Cell. New York: Garland, 2007, pp. 880$883.487-490$ 